\section{A flexible, multiple-graph plotting program for interactive use}

\author{
GARY KAMEN \\ Department of Physical Education, Indiana University \\ Bloomington, Indiana 47405
}

The program described here can plot several graphs on a single page suitable for journal publication. Several of the key features of the program are shown in Figure 1. The user can select dashed, dotted, or solid lines with as many as 30 points on the $x$-axis. Minor tic marks can be selected on the $y$-axis, and several types of options may be used for the $x$-axis. If several graphs share the same $x$-axis, it is possible to suppress the plot of the $\mathrm{x}$-axis. Standard deviation or standard error bars may be plotted in several different formats. Finally, the user has control over the type of labels or comments to be placed at any place on the graph and the size of each character in any part of the graph. The program has been used for figures published in several American Psychological Association (APA) and non-APA journals (Clarkson, Kamen, \& Kroll, 1980; Kamen, Kroll, Clarkson, \& Zigon, 1981; Kroll, Clarkson, Kamen, \& Lambert, 1980).

Input. The parameters specific to each graph (or set of graphs) are entered via an interactive routine that is largely self-explanatory. The user is asked to enter such information as the number of graphs to be plotted, specifications for the $x$-axis and the $y$-axis, the number of dashed lines, and so on. The interactive program creates a disk file that contains all of these parameters in an easy-to-read format. The data for each graph are stored in a separate disk file. In this way, extremely complicated graphs can be modified without repeating the entire interactive sequence.

Output. The program prints out the raw data file as well as the parameters selected for the graph. The user can then compare the plotted output with the selected parameters to optimize the quality of the graph.

Language and Computer. The program is written in standard FORTRAN IV using the CDC $6600 \mathrm{com}$ puter. Several plotting subroutines supplied by California Computer Products, Inc. (Calcomp) were used or modified for use. The program should be readily adaptable to
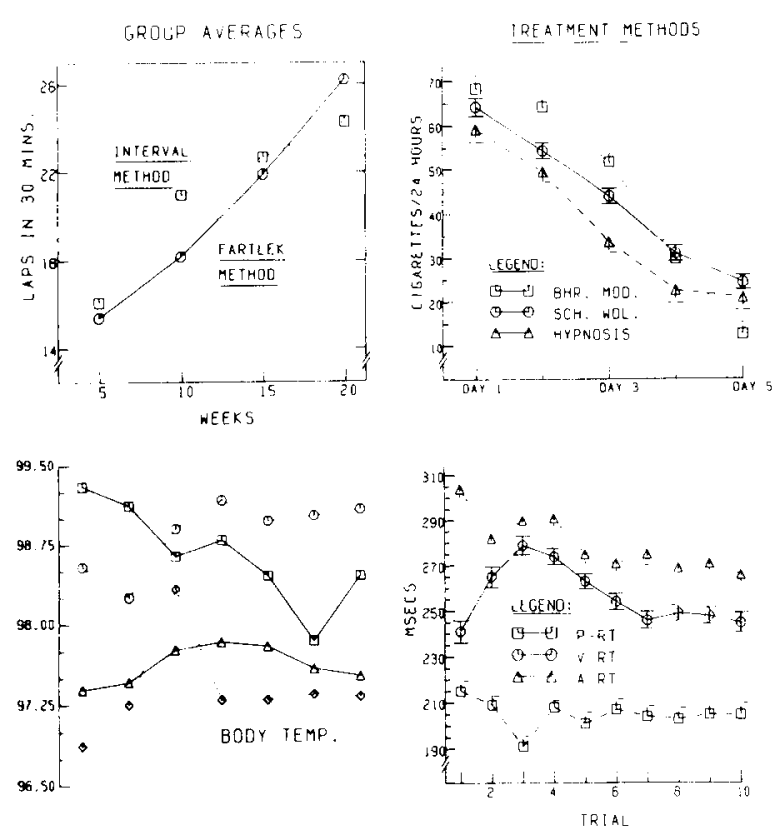

Figure 1. These graphs demonstrate many of the options available in the plotting program.

any computer system that has the Calcomp software available and a minimum $32 \mathrm{~KB}$ of core space.

Documentation and Availability. A listing of the plotting program and detailed step-by-step documentation, as well as sample plots, may be obtained from the author at Indiana University, Department of Physical Education, Bloomington, Indiana 47405.

\section{REFERENCES}

Clarkgon, P. M., Kamen, G., \& Kroll, W. Knee and ankle extension isometric endurance and muscle composition in power and endurance athletes. Journal of Sports Medicine and Physical Fitness, 1980, 20, 255-264.

Kamen, G., Kroll, W., Clakkson, P. M., \& Zigon, S. T. Fractionated reaction time in power-trained and endurance-trained athletes under conditions of fatiguing isometric exercise. Journal of Motor Behavior, 1981, 13, 117-129.

Kroll, W., Clarkson, P. M., Kamen, G., \& Lambert, J. Muscle fiber type composition and knee extension isometric strength fatigue patterns in power and endurance-trained males. Research Quarterly for Exercise and Sport, 1980, 51, 323-333.

(Accepted for publication March 13, 1982.) 